\title{
Metodología de diseño aplicada en dispositivo de pruebas en pistones
}

\section{Design methodology applied in piston testing device}

\author{
RAMÍREZ-CRUZ, José Luis†๋*, JIMÉNEZ-RABIELA, Homero, VÁZQUEZ-GONZÁLEZ, Benjamín \\ y GARCÍA-SEGURA, Pedro
}

Universidad Autónoma Metropolitana. Unidad Azcapotzalco. División CBI. Departamento de Energía

ID $1^{\mathrm{er}}$ Autor: José Luis, Ramírez-Cruz / ORC ID: 000-0003-0762-2630, Researcher ID Thomson: G-3405-2019, CVU CONACYT ID: 921268

ID $1^{\text {er }}$ Coautor: Homero, Jiménez-Rabiela / Researcher ID Thomson: S-2299-2018, CVU CONACYT ID: 123386

ID $2^{\text {do }}$ Coautor: Benjamín, Vázquez-González / ORC ID: 0000-0002-9030-5662, Researcher ID Thomson: S-2417-2018, CVU CONACYT ID: 25749

ID $3^{\text {er }}$ Coautor: Pedro, García-Segura / ORC ID: 0000-0003-4947-084X, Researcher ID Thomson: S-2360-2018, CVU CONACYT ID: 371233

DOI: $10.35429 / J I D .2020 .11 .4 .14 .18$

Recibido 23 de Julio, 2020; Aceptado 12 de Octubre, 2020

\begin{abstract}
Resumen
Como parte del desarrollo y fabricación de pistones para motores utilizados por la industria automotriz, se realizan una serie de ensayos de laboratorio que incluyen: Pruebas mecánicas, metalográficas y análisis químico del material, verificación dimensional, del peso final y de los acabados de los pistones terminados, pruebas en el dinamómetro de motor del producto terminado, etc. El departamento de desarrollo tecnológico de una empresa fabricante de pistones automotrices, como parte del proceso de aseguramiento de calidad de sus productos, ha establecido la necesidad de evaluar la resistencia a la compresión y a la fatiga de pistones, tanto a temperatura ambiente como a la temperatura normal de operación de un motor de combustión interna. El equipo propuesto para realizar estas pruebas es una máquina servo hidráulica, la cual tiene capacidad de aplicar carga dinámica y carga estática, con las cuales se puede simular cargas extremas de operación de los pistones. Por este motivo, la meta de diseño de este trabajo es el diseñar un dispositivo con el cual se pueda sujetar las probetas de prueba (pistones) en la máquina, para poder realizar pruebas de fatiga y compresión y en cual, se pueda calentar el pistón a la temperatura requerida de prueba.
\end{abstract}

Pistón, Fatiga, Simulación

\begin{abstract}
:
As part of the development and manufacture of engine pistons used by the automotive industry, a series of laboratory tests are carried out that include: Mechanical, metallographic and chemical analysis of the material, dimensional verification, final weight and piston finishes finished, tests on the engine dynamometer of the finished product, etc. The technological development department of an automotive piston manufacturing company, as part of the quality assurance process of its products, has established the need to assess the compressive and fatigue resistance of pistons, both at room temperature and at temperature normal operation of an internal combustion engine. The equipment proposed to carry out these tests is an hydraulic servo machine, which has the capacity to apply dynamic load and static load, with which extreme piston operating loads can be simulated. For this reason, the design goal of this work is to design a device with which the test specimens (pistons) can be held in the machine, in order to perform fatigue and compression tests and in which, the heat can be heated piston at the required test temperature.
\end{abstract}

Piston, Mechanical test, Fatigue, Simulation, Design

Citación: RAMÍREZ-CRUZ, José Luis, JIMÉNEZ-RABIELA, Homero, VÁZQUEZ-GONZÁLEZ, Benjamín y GARCÍASEGURA, Pedro. Metodología de diseño aplicada en dispositivo de pruebas en pistones. Revista del Diseño Innovativo. 2020, 4-11: 14-18

\footnotetext{
*Correspondencia al Autor (Correo electrónico: rcjl@azc.uam.mx)

$\dagger$ Investigador contribuyendo como primer autor.
} 


\section{Introducción}

El INEGI dio a conocer los registros administrativos de la industria automotriz de vehículos ligeros que se desarrolla en el país, provenientes de 21 empresas afiliadas a la Asociación Mexicana de la Industria Automotriz, A.C. (AMIA), como son las ventas al público en el mercado interno, producción y exportaciones para el mes de septiembre del presente año.

Aproximadamente el $63 \%$ de los vehículos ligeros que se producen en México es exportado al mercado de Estados Unidos [1] y, por lo tanto, las regulaciones y preferencias del consumidor en éste mercado, afectan tanto a los vehículos que se producen en México, como a las partes y componentes de los mismos incluyendo el sector de pistones originales y de refacción.

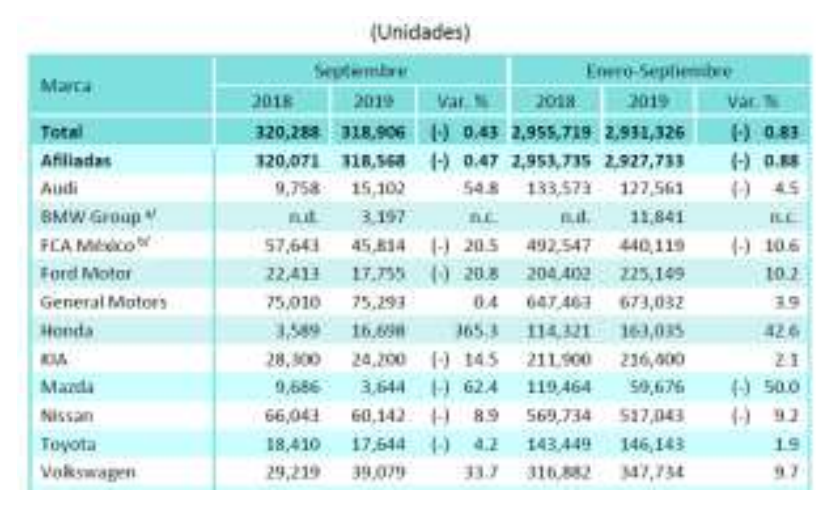

Figura 1 Producción total de vehículos ligeros Fuente: Tomado de www.inegi.org.mx

Cabe aclarar que la estadística en la producción de pistones del mercado de refacciones, no se incluye la producción de pistones utilizados en la producción de motores, por lo tanto la producción total de pistones en México para el año 2018 alcanzó alrededor de 19 millones de piezas. Las tendencias en la fabricación de los motores a gasolina para la industria automotriz se pueden englobar en los siguientes requerimientos:

\footnotetext{
- Disminuir las emisiones contaminantes

- Aumentar el rendimiento de combustible

Soportar:

- Elevadas temperaturas $\left(>350^{\circ} \mathrm{C}\right)$.

- Elevadas presiones en la cámara de combustión (Figura 5)

- Altas revoluciones por minuto (> 5000).

- Procesos de desgaste.
}

- Procesos de corrosión.

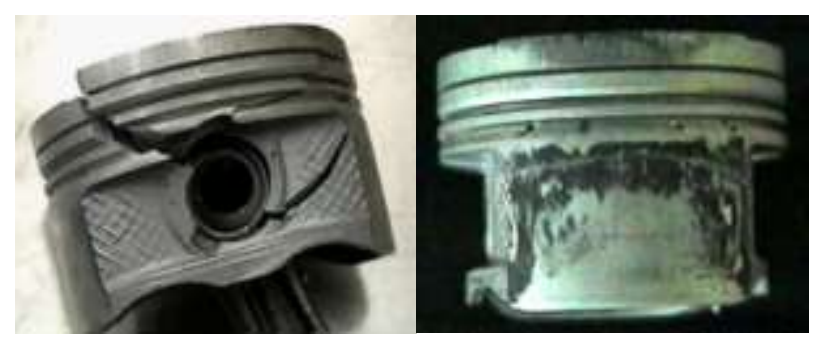

Figura 2 Daños en pistones por fatiga y por desgaste Fuente: Elaboración Propia

Los pistones son uno de los componentes más complejos entre todos los componentes de la industria automotriz u otros, siendo estos la parte más importante de un motor. Debido a la cantidad de ciclos y a las altas temperaturas, se han realizo estudios de fatiga en diferentes casos, simulando estas condiciones. En la figura 3 permite ver el estado de esfuerzos de un pistón bajo una presión de trabajo.

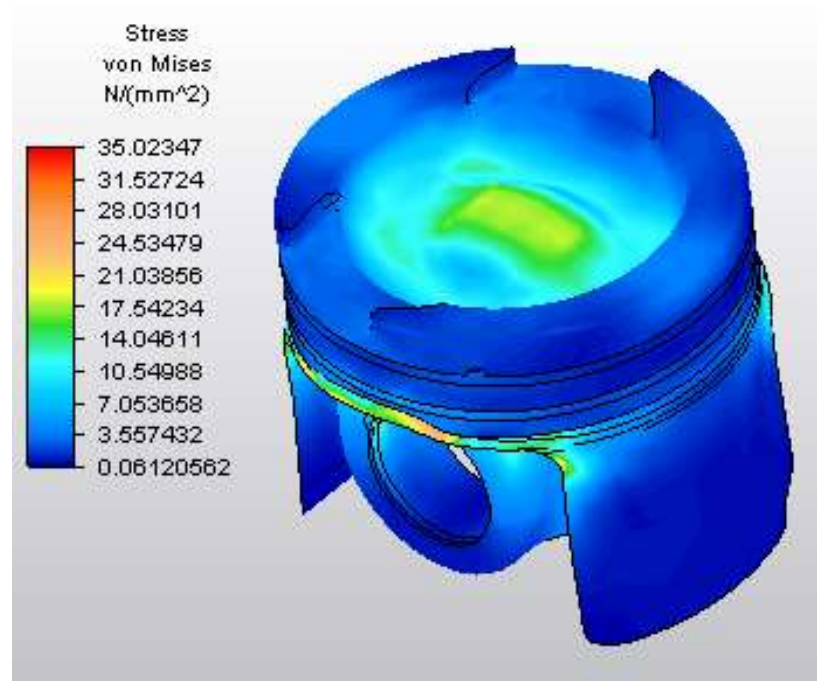

Figura 3 Simulación de esfuerzos en un pistón Fuente: Elaboración Propia

El departamento de desarrollo tecnológico de un fabricante de pistones automotrices, como parte del proceso de aseguramiento de calidad de sus productos, ha establecido la necesidad de evaluar la resistencia a la compresión y a la fatiga de los pistones que desarrolla y fabrica, tanto a temperatura ambiente como a la temperatura normal de operación de un motor de combustión interna. El equipo propuesto para realizar estas pruebas es la maquina servo hidráulica, el cual tiene capacidad de para aplicar carga dinámica y carga estática, con las cuales se puede simular cargas extremas de operación de los pistones.

RAMÍREZ-CRUZ, José Luis, JIMÉNEZ-RABIELA, Homero, VÁZQUEZ-GONZÁLEZ, Benjamín y GARCÍA-SEGURA, Pedro. Metodología de diseño aplicada en dispositivo de pruebas en pistones. Revista del Diseño Innovativo. 2020 
El presente trabajo tiene como propósito mostrar el diseño de un dispositivo con el cual se pueda sujetar las probetas de prueba (pistones) en una máquina de pruebas mecánicas que se usará para poder realizar pruebas de fatiga y compresión y que en éste, se pueda calentar el pistón a la temperatura de prueba requerida.

\section{Requerimientos de diseño}

La actividad de diseño empieza con el reconocimiento y la determinación de una necesidad o deseo relativo a un producto, servicio, o sistema, y a la posibilidad de satisfacer esa necesidad.

Los requerimientos de las características del dispositivo de pruebas, fueron generados por entrevistas personales y por vía telefónica, las cuales se han agrupado y se describen a continuación:

\section{Requerimientos obligatorios}

- Que permita evaluar la resistencia a la fatiga de un pistón terminado.

- Que permita evaluar la resistencia a la compresión de un pistón terminado.

Que el dispositivo realice la prueba de manera confiable.

Que el dispositivo se acople a la máquina Instron.

Que sean seguras las maniobras de montaje y desmontaje.

Que no se generen condiciones inseguras durante la prueba.

Que se desarrolle en un plazo máximo de 8 meses.

- Que el costo del dispositivo no sobrepase $\$ 7,500.00$ dolares.

\section{Requisitos deseables}

- Que reproduzca fenómenos de desgaste en el pistón de prueba.

- Que se puedan probar pistones diferentes.

- Que simule la temperatura de operación normal del pistón.

- Que el armado del dispositivo sea fácil.

- Que sea fácil el montaje y desmontaje del dispositivo de pruebas en la máquina de pruebas.
- Que sea fácil el montaje y desmontaje de los pistones en el dispositivo de pruebas.

La información concerniente al problema identificado se convierte en la base para una proposición del problema, la que puede consistir en información, presentada para su consideración formal.

\begin{tabular}{|c|c|c|c|c|}
\hline & & & $\begin{array}{r}\text { Met } \\
\text { dis }\end{array}$ & $\begin{array}{l}\text { as de } \\
\text { eño }\end{array}$ \\
\hline \# & Variable & & $\mathrm{P}-1$ & $\mathrm{P}-2$ \\
\hline 1 & Carga máxima. & KN & 400 & 180 \\
\hline 2 & Carga mínima. & KN & 0 & 80 \\
\hline 3 & $\begin{array}{l}\text { Frecuencia de aplicación de } \\
\text { carga. }\end{array}$ & $\mathrm{Hz}$ & 1 & 10 \\
\hline 4 & Temperatura de prueba. & ${ }^{\circ} \mathrm{C}$ & $\begin{array}{r}25- \\
350 \\
\end{array}$ & $\begin{array}{r}25- \\
350 \\
\end{array}$ \\
\hline 5 & $\begin{array}{l}\text { Porcentaje de área de } \\
\text { contacto con la cabeza del } \\
\text { pistón. }\end{array}$ & $\%$ & 100 & 100 \\
\hline 6 & Longitud del dispositivo. & $\mathrm{mm}$ & 300 & 300 \\
\hline 7 & Ancho del dispositivo. & $\mathrm{mm}$ & 300 & 300 \\
\hline 8 & Altura del dispositivo. & $\mathrm{mm}$ & 700 & 700 \\
\hline 9 & Diámetro del pistón. & $\mathrm{mm}$ & $\begin{array}{l}55- \\
100\end{array}$ & $\begin{array}{l}55- \\
100\end{array}$ \\
\hline $\begin{array}{l}1 \\
0\end{array}$ & Altura del pistón. & $\mathrm{mm}$ & $\begin{array}{r}40- \\
60\end{array}$ & $\begin{array}{r}40- \\
60\end{array}$ \\
\hline $\begin{array}{l}1 \\
1\end{array}$ & Carrera del pistón. & $\mathrm{mm}$ & $\begin{array}{c}60- \\
100\end{array}$ & $\begin{array}{r}60- \\
100\end{array}$ \\
\hline $\begin{array}{l}1 \\
2\end{array}$ & $\begin{array}{l}\text { Peso máximo de las partes } \\
\text { del dispositivo. }\end{array}$ & $\mathrm{kg}$ & $<15$ & $<15$ \\
\hline $\begin{array}{l}1 \\
3\end{array}$ & $\begin{array}{l}\text { Peso total máximo del } \\
\text { dispositivo. }\end{array}$ & $\mathrm{kg}$ & $<50$ & $<50$ \\
\hline 1 & Personal necesario. & Cantidad & 3 & 3 \\
\hline 1 & Herramientas necesarias. & Cantidad & 4 & 4 \\
\hline
\end{tabular}

Tabla 1 Metas de diseño. P-1 Prueba de compresión. P-2 Prueba de fatiga

Fuente: Elaboración Propia

\section{Definición del modelo funcional}

Una función representa el papel que desempeña un elemento o un conjunto completo. Una función del producto es una declaración de una relación clara y reproducible entre la entrada disponible y la salida deseada de un producto, independiente de cualquier forma particular.

A continuación, se describe el modelo funcional del Dispositivo para pruebas de fatiga y compresión, para pistones de motores a gasolina. 
Las funciones de servicio y su clasificación del dispositivo para pruebas de fatiga y compresión, para pistones de motores a gasolina, se indican en la tabla $2 \mathrm{y}$, en la figura 4 se muestra la función global de servicio que se relacionan con cada uno de las funciones de servicio.

\begin{tabular}{|l|l|}
\hline \multicolumn{2}{|c|}{ Clave } \\
\hline A1 & Montar el pistón en el dispositivo \\
\hline A2 & Colocar interfaz entre pistón y dispositivo \\
\hline A3 & $\begin{array}{l}\text { Calentar pistón e interfaz a la temperatura de } \\
\text { prueba }\end{array}$ \\
\hline A4 & $\begin{array}{l}\text { Transferir cargas de fatiga y compresión al } \\
\text { pistón }\end{array}$ \\
\hline A5 & Fracturar el pistón por fatiga o compresión \\
\hline A6 & Desmontar el pistón de fracturado \\
\hline
\end{tabular}

Tabla 2 Funciones de servicio y su clasificación Fuente: Elaboración Propia

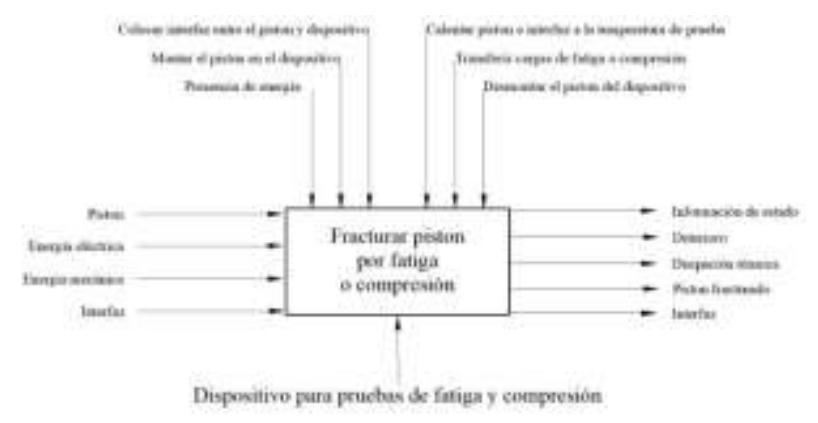

Figura 4 Descripción de la función global Fuente: Elaboración Propia

\section{Diseño de detalle}

La actividad de diseño empieza con el reconocimiento y la determinación de una necesidad o deseo relativo a un producto, servicio, o sistema, y a la posibilidad de satisfacer esa necesidad.

Una vez que definidas las características que se deben de cumplir y como es que se cubrirán, a continuación pasamos a la etapa de diseño para ver obtener de una manera visual las características propuestas.

En este momento se diseñan cuidadosamente todas las partes, basándose en su resistencia y función. Una vez que esté bien definido el producto y siempre teniendo en cuenta los costos, este debe construirse en un lapso mínimo de tiempo.

En la actualidad mediante algún programa computacional especializado es posible obtener un modelo virtual muy cercano a la realidad.

ISSN-2523-6830

ECORFAN $^{\circledR}$ Todos los derechos reservados

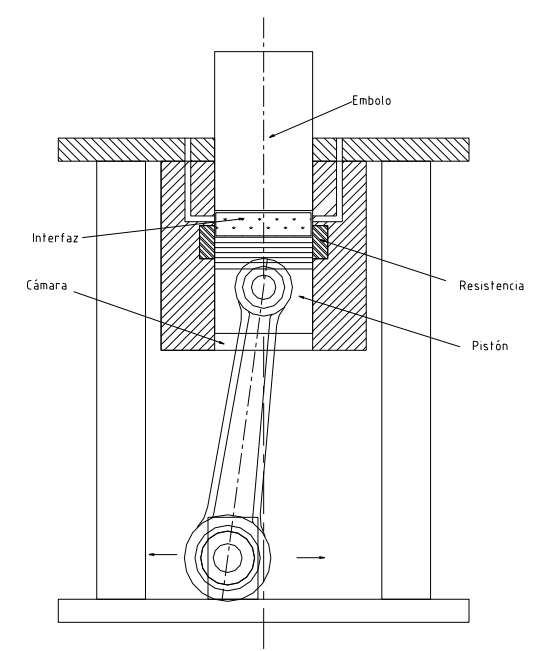

Figura 5 Esquema del concepto de diseño del dispositivo para pruebas de fatiga y compresión Fuente: Elaboración Propia

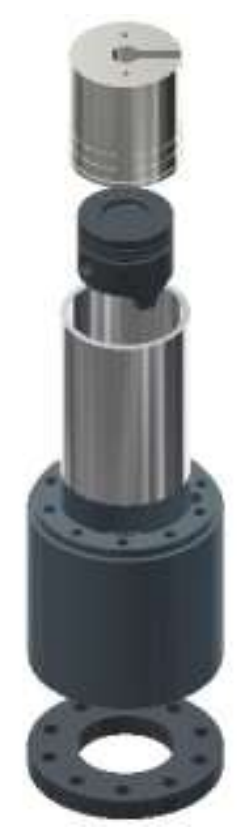

Figura 6 Despiece del ensamble virtual del dispositivo para pruebas de fatiga y compresión para pistones de motores a gasolina

Fuente: Elaboración Propia

Para hacer esto, se utilizó software para dibujo mecánico en 3D y para el modelado de piezas y conjuntos. En la figura 6, se encuentra la propuesta que resultó del análisis con la metodología de diseño.

El resultado del diseño de las características del dispositivo se muestra en la figura 7.

Este dispositivo contiene las características descritas anteriormente y sería el más adecuado para fabricar y colocarlo en máquina. 


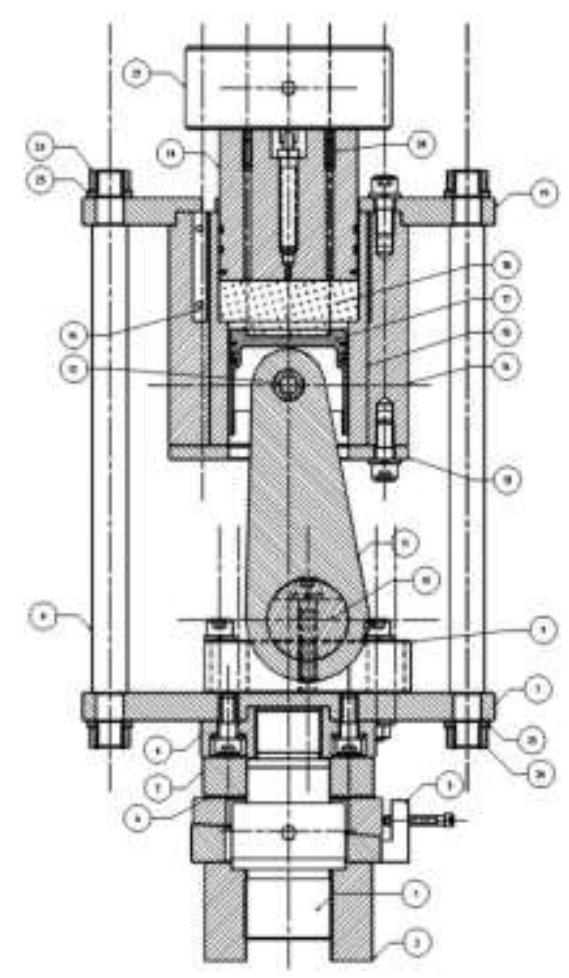

Figura 7 Dibujo de ensamble virtual del dispositivo para pruebas de fatiga y compresión para pistones de motores a gasolina

Fuente: Elaboración Propia

\section{Conclusiones}

El artículo propuesto hace referencia al desarrollo de una metodología de diseño que aplica para cualquier tipo de máquina, equipo o dispositivo que se desee desarrollar.

El proyecto cumple satisfactoriamente con las especificaciones establecidas en el diseño, como lo son la capacidad de la máquina y su rendimiento.

Se concluye que la metodología es una herramienta que permite enfocar los esfuerzos con el fin de comprender las necesidades del cliente y traducirlos a términos de diseño de ingeniería, con lo cual se asegura el éxito del proyecto.

\section{Referencias}

Chevalier A. (1997), Dibujo Industrial, Limusa, México.

Hamrock Bernard J. (2000), Elementos de máquinas, McGraw-Hill, México.

https://www.inegi.org.mx/contenidos/saladepre nsa/notasinformativas/2019/rm_raiavl/rm_raiav 12019_10.pdf
Otto K. (2001). Product design. Prentice-Hall. U.S.A. 